\title{
Comparing Parametric and Nonparametric Statistical Methods for Studying the Velocity Distributions of Population I Stars
}

\author{
F. ARENOU \\ URA D0335 CNRS, Observatoire de Paris-Meudon \\ F-92195 Meudon, France \\ M. L. BOUGEARD \\ URA 1125 CNRS: I.E.R.S., Observatoire de Paris \\ 61 Av. de l'Observatoire, F-75014 Paris, France
}

\begin{abstract}
This Paper investigates population I star samples from the viewpoint of the velocity distributions, each being viewed as a mixture. To obtain information on the class centers, clustering techniques are first applied. Secondly, we use a parametric maximum likelihood formulation that we solve by Redner-Walker's E.M. algorithm. The obtained results are compared and discussed from an astrophysical viewpoint.
\end{abstract}

Key words: - velocity distribution - mixtures - clusters - E.M. algorithm.

\section{INTRODUCTION}

The stellar distribution in Our Galaxy is a function of several variables and it is quite known that Stellar Kinematics play a major role in studies of the galactic structure. Adopting the usual description of the observed space velocity of a star with respect to the Sun in terms of a three dimensional vector $(U, V, W)$, the observed distribution in each component has been long assumed as nearly gaussian. Gómez et al. (1990) defined particular homogenous Population I samples that are nearly complete up to a limiting magnitude of stars located in the solar neighborhood at a distance smaller than 200 parsecs from the Sun, covering ages up to about $610^{8}$ years. They proved that the multivariate velocity densities can be viewed as a mixture of populations related to different star formation bursts. Here, our aim is to compare different statistical methods of separation of the components by focusing our attention on their A2 V-sample (

In Section 2 nonparametric clustering techniques are applied. Then, assuming a mixture of multivariate Gaussians in Section 3, we use a maximum likelihood 
approach that we solve numerically by the recent and powerful E.M. algorithm. The obtained results are compared and discussed.

\section{SEPARATING COMPONENTS: NONPARAMETRIC METHODS}

\subsection{From an univariate approach to a multivariate one}

Although $U, V$ are highly correlated, it becomes apparent by using KolmogorovSmirnov univariate tests of normality, that, among $U, V, W$, only $U$ seems nearly gaussian. Moreover the $U$ distribution is assymetric and on a $(U, V)$ graph, one can guess a mixture of at least two groups. So, an analysis will be performed on the global $(U, V, W)$ distribution.
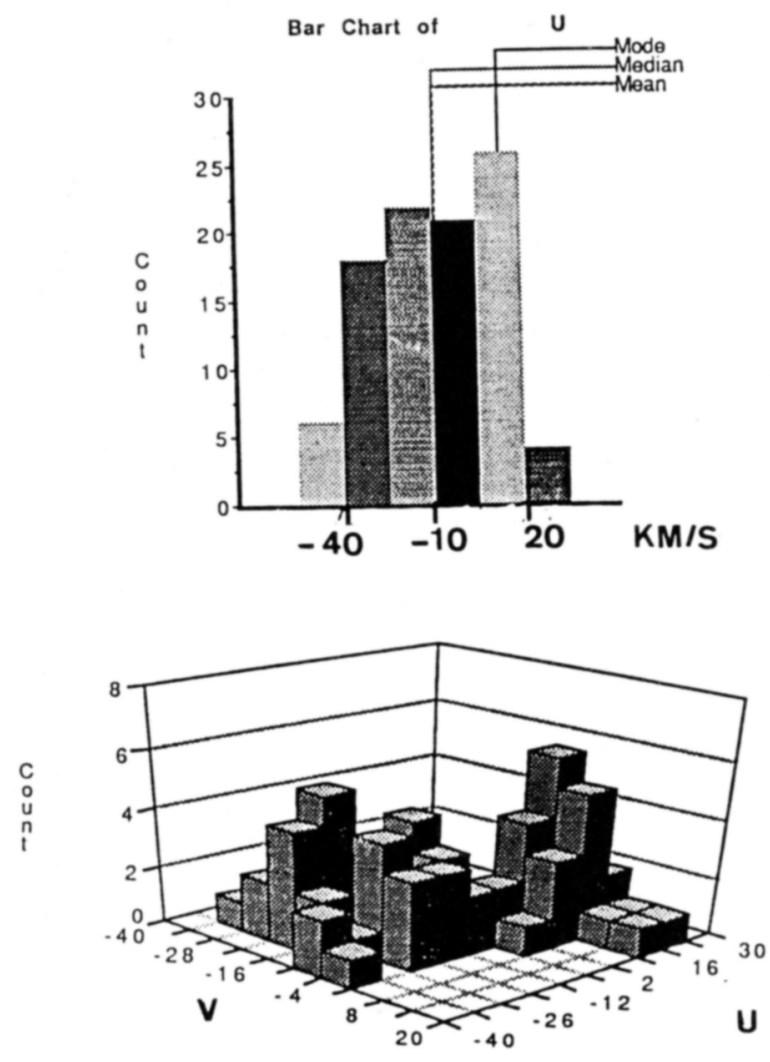

Fig.1. On the top, the $U$-distribution, on the bottom the bidimensional $(U, V)$ one 


\subsection{Method}

Several hierarchical clustering approaches are available. Here, we apply the "Nearest Neighbour chain" NN-algorithm that classifies observations into groups using a nonparametric method (Hand, 1981).

\subsection{Results}

We obtain a natural partition into two components $\left(A_{c}, B_{c}\right)$ that is shown on Fig.2 drawn in the U-V galactic plane. The centroid of each class is given by:

$$
\begin{array}{lllll}
A_{c}: & \bar{U}=-22.5 & \bar{V}=-14.1 & \bar{W}=-6.6 & (n=57 \text { stars among } 97) \\
B_{c}: & \bar{U}=+10.3 & \bar{V}=-0.4 & \bar{W}=-7.6 & (n=40 \text { stars among } 97)
\end{array}
$$

We have noticed that, in the present case, a non hierarchical classification based on aggregation around moving centers leads to similar results.

\section{PARAMETRIC ESTIMATION}

\subsection{Statistical formulation}

In order to apply a maximum likelihood method, we assume in the following that the velocity distribution $f(x), x=(U, V, W)$ of the A2 V-sample can be realistically represented as a convex combination of Gaussians $\Phi_{i}, i=1-k$ with mean $m_{i}$, variance $\Sigma_{i}$, respective weights $p_{i}$ :

$$
f(x, \theta)=p_{1} \Phi_{1}\left(x \mid m_{1}, \Sigma_{1}\right)+\ldots+p_{k} \Phi_{k}\left(x \mid m_{k}, \Sigma_{k}\right)
$$

Methods for estimating the unknown parameters $\theta=\left(p_{i}, m_{i}, \Sigma_{i}, i=1-k\right)$ have been and are still widely discussed in the statistical litterature. As it is well known, some difficulties arise in the case of overlapping in the components and when the sample size is "statistically" small (the present case).

\subsection{The Redner-Walker (1984) E.M. algorithm}

In order to maximize the log-likelihood function $L(\theta)$ of the sample, we use the Redner-Walker E.M. algorithm that is known to converge and to preserve the parameter constraints compared to the Newton method. Recall that the updating rule is based on the evaluation at step $E$ (expectation step) of an ad-hoc estimate $p_{i j}$ of the posterior probability that an observation $x_{j}$ belongs to the $i$ th group, given the current estimate of $\theta$. Then, step $M$ (maximization) updates $\theta$ with reference to this $p_{i j}$ (see formulation in: Bougeard and Arenou, 1990).

\subsection{Results and interpretation}

Several independent runs have to be performed since the resulting likelihood surface is littered with singularities that potentially occur on the boundary of the parameter 
space. For the A2 V sample under consideration here, a 2-mixture solution $(A, B)$ with respective weights 0.64 and 0.36 has been found as significant. We obtain as estimates of the mean values and of the diagonal of the variance matrix:

$A:\left(m_{U^{\prime}}, m_{V}, m_{W}\right)=(-20.8,-14.3,-6.8) \quad\left(\sigma_{U}, \sigma_{V}, \sigma_{W}\right)^{2}=(13.3,10.2,8.7)^{2}$

$B:\left(m_{U}, m_{V}, m_{W}\right)=(+11.4,+1.7,-7.4) \quad\left(\sigma_{V}, \sigma_{V}, \sigma_{W}\right)^{2}=(7.1,5.8,7.1)^{2}$

Moreover the $p_{i j}$ permit a classification of the stars in each group that is shown on Fig.2.
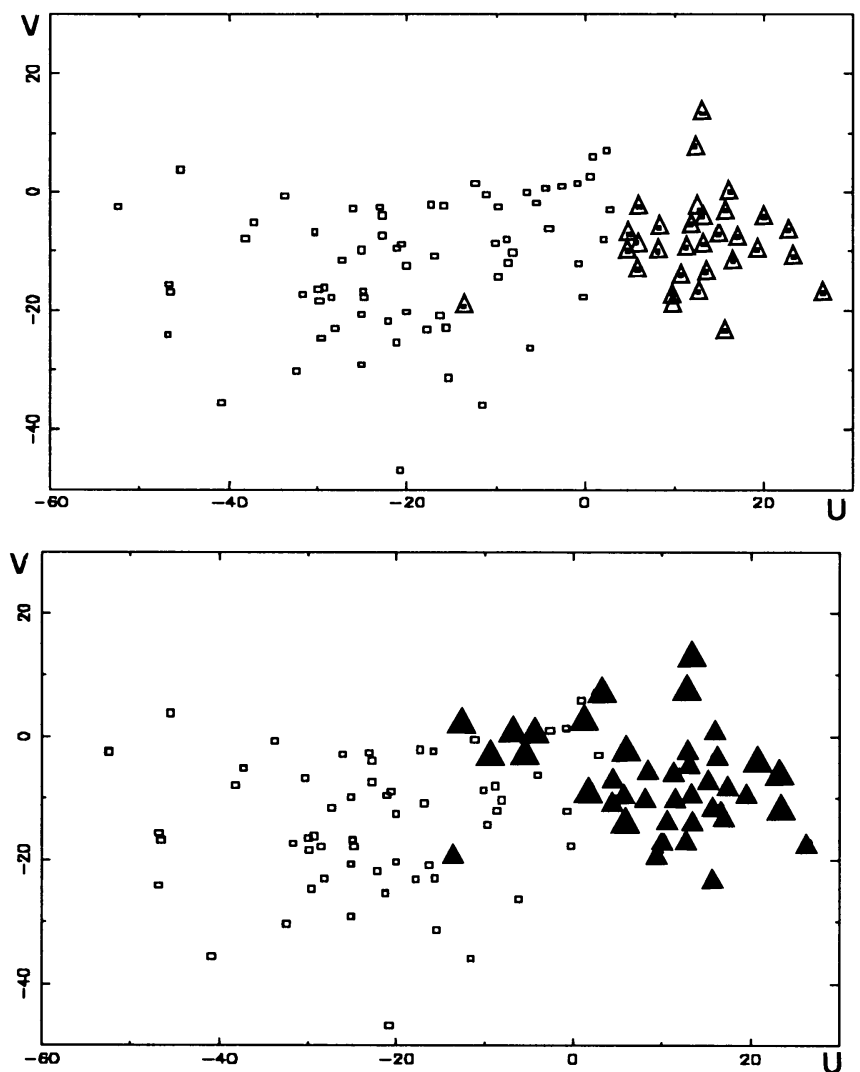

Fig.2. Visualisation of the 2-mixture in the galactic plane: on the top, the nonparametric clusters, on the bottom the E.M. groups 
It can be noticed that the above parametric results are in agreement with those of the clustering method. This supports the hypothesis that this sample is in fact a mixture of two populations.

Gómez et al. (1990) interpret each group as formed in a particular star burst. Moreover, they assume that each burst also produced open clusters. At this time, the individual ages of the stars in the sample under study are not available; the age is clearly the discriminant variable which could support this interpretation in terms of star formation bursts. Nevertheless, we can notice that the above statistical estimations of the $A$ group mean values agree with the velocity components of the IC 2391 young cluster and the $B$ group with $\mathrm{U}$ Ma cluster that is older.

\section{CONCLUSION}

In this Paper we have been concerned with the problem of separating components of a mixture that is a problem of importance in Astronomy (study of velocity distributions in terms of star formation bursts,...). Different methods were examplified in application to an A2 V-sample. Further statistical investigations are to be found in a forthcoming paper. Nevertheless, larger difficulties were encountered for the analysis of samples with smaller size. Recall that for a 2-component mixture in three dimensions, the estimation of 1 (proportion) +3 (mean-values) +6 (covariance-terms) $=$ 10 parameters are needed.

The results will be improved as soon as a better knowledge of the star velocities will be obtained, allowing the use of larger samples.

\section{REFERENCES}

Bougeard M.L., Arenou F.,1990: in Errors, Biais, Uncertainties in Astronomy, Cambridge Press, 277-280

Bougeard M.L., Arenou F., Gómez A.E., 1989, I.S.I. 49th Session

Gómez A.E., Delhaye J., Grenier S., Jaschek C., Arenou F., Jaschek M., 1990, Astron. Astrophys. 236, 95

Hand D.J., 1981, Discrimination and classification, N. York, J. Wiley

Redner, Walker, 1984, S.I.A.M., $26 \mathrm{n}^{\circ} 2,195-239$ 